\section{International Scientific Journal Theoretical \& Applied Science}

p-ISSN: 2308-4944 (print) e-ISSN: 2409-0085 (online)

Year: $2018 \quad$ Issue: 09 Volume: 65

Published: 21.09.2018 http://T-Science.org
Denis Nikolaevich Drozdov

Ph.D. in Biological Sciences,

Associate Professor

Gomel State University named Skaryna

L.S. Shahlenkova

Graduate student

Gomel State University named Skaryna

lilia54321@mail.ru

SECTION 11. Biology. Ecology. Veterinary.

UDC 612.117 + 612.017.1 + 612.014.4

\title{
AGE DYNAMICS OF ELECTRICAL CARDIAC AXIS IN HEALTHY PEOPLE IN CONNECTION WITH CHANGE SOMATOMETRIC STATUS DRUG PERIODS OF POSTNATAL ONTOGENESIS
}

\begin{abstract}
The relationship between the somatometric status and the age dynamics of the electrical axis of the heart is shown. To study this dependence, an experimental material was collected, which allowed the development of a somatotype determination model taking into account age dynamics of the angle of inclination of the electric axis of the heart. As a result of the study, the limits of the alpha angle variation of the electrical axis of the heart for different ages and somatotype were determined. The age-related limits of the values of the angle of inclination of the electric axis of the heart, obtained with the help of the model, can be used for comparison as the proper values with the data of the electrocardiography analysis.

Key words: electrical cardiac axis, somatotype, the somatometric status physique, Pignier index

Language: Russian

Citation: Drozdov DN, Shahlenkova LS (2018) AGE DYNAMICS OF ELECTRICAL CARDIAC AXIS IN HEALTHY PEOPLE IN CONNECTION WITH CHANGE SOMATOMETRIC STATUS DRUG PERIODS OF POSTNATAL ONTOGENESIS. ISJ Theoretical \& Applied Science, 09 (65): 75-81.

Soi: http://s-o-i.org/1.1/TAS-09-65-13 Doi: crossef https://dx.doi.org/10.15863/TAS.2018.09.65.13

\section{ВОЗРАСТНАЯ ДИНАМИКА ЭЛЕКТРИЧЕСКОЙ ОСИ СЕРДЦА У ЗДОРОВЫХ ЛЮДЕЙ В СВЯЗИ С ИЗМЕНЕНИЕМ ИХ СОМАТОМЕТРИЧЕСКОГО СТАТУСА В РАЗНЫЕ ПЕРИОДЫ} ПОСТНАТАЛЬНОГО ОНТОГЕНЕЗА
\end{abstract}

Аннотация: В работе показана связь соматометрического статуса с возрастной динамикой электрической оси сердия. Для исследования этой зависимости собран экспериментальный материал, который позволил разработать модель определения соматотипа с учетом возрастной динамики угла наклона электрической оси сердиа. В результате исследования определены границы варьирования угла альфа электрической оси сердца для разного возраста и соматотипа. Полученные с помощью модели возрастные границы значений угла наклона электрической оси сердия, могут быть использованы для сопоставления в качестве должных величин с данными ЭКГ-анализа.

Ключевые слова: электрическая ось сердия, соматотип, телосложение, индекс Пинье

\section{Введение.}

Возрастные изменения в работе сердца выражаются в динамике показателей электрокардиограммы. Среди этих показателей особый интерес представляет возрастная динамика электрической оси сердца (ЭОС). В ряде случаев крайнее правое и крайнее левое положение ЭОС служит дополнительным диагностическим признаком нарушения проводимости и состояния гипертрофии миокарда предсердий, желудочков, а также блокады ножек пучка Гиса. В настоящее время в доступной научной литературе представлены данные о вариации положения ЭОС, связаны с той или иной формой патологии сердца. Например, гипертрофией миокарда спортсменов [1, 2], алкогольной интоксикацией сердца [3], гипертрофической кардиомиопатией (ГКМП) обусловленной генетическими нарушениями [4] и др. Однако вариация положения ЭОС зависит не только от патологических, но и от морфологических изменений, как в самом сердце, так и в целом в организме человека. Среди возрастных изменений, происходящих на уровне организма, можно выделить колебания мышечной и жировой массы тела, а также изменения, 
возникающие в результате перераспределения гемодинамической нагрузки из-за этих колебаний.

У здорового человека угол наклона ЭОС на фронтальную плоскость или на ось первого стандартного отведения (угол альфа), располагается в сегменте от $0^{\circ}$ до $+90^{\circ}$ (нормограмма) и изредка выходит за эти пределы [5]. В норме угол наклона ЭОС соответствует углу наклона и ориентации анатомической оси сердца. Отклонение в направление горизонтального положения, когда угол альфа с фронтальной плоскостью составляет от $0^{\circ}$ до $29^{\circ}$ (левограмма) встречается у здоровых людей с гиперстеническим типом телосложения (дигестивный тип), отклонение в направление вертикального положения попадает в сегмент от $90^{\circ}$ до $75^{\circ}$ (правограмма) и характерно для здоровых людей с астеническим типом телосложения. В возрастном аспекте в норме у детей грудного и дошкольного возраста (до 7 лет) имеет место отклонение ЭОС вправо. У детей школьного возраста и подростков, вплоть до окончания пубертатного периода (от 7 до 15-17 лет) устанавливается нормальное положение ЭОС, которое сохраняется до 60 лет, у людей старше 60 лет доминирует левограмма. В литературных источниках указывается, что в течение жизни в динамике положения ЭОС наблюдается тенденция отклонения этого показателя из вертикального в горизонтальное положение, которую обуславливают возрастные изменения пропорций тела [6].

\begin{tabular}{ccr} 
Изучение & возрастной & \multicolumn{2}{c}{ изменчивости } \\
пропорций тела человека & и влияния
\end{tabular} соматотипических особенностей на физическое развитие и физиологический статус, имеет достаточно большую историю и подробно рассмотрены в работах отечественных и зарубежных ученых (Л.Ф. Яроцинский, К.О. Фалькович, В.В. Бунак, П. Н. Башкиров, У. Шелдон, Л. Картер и др.). В работах Русалова (1979) получены убедительные экспериментальные доказательства того, что соматотип действует как единый фактор. В ряде работ, М.С. Маслова, М.В. Черноруцкого, В.Н. Шевкуненко, установлена связи между весоростовыми показателями и сенситивностью разных систем организма человека. Анализ динамики этих признаков в онтогенезе показал, что в период полового созревания у подростков дигестивного и астенического типа наблюдается более раннее прекращение роста тела в длину, что обусловлено отличным от нормостенического типа, уровнем секреции половых гормонов. В работах М. В. Черноруцкого показано, что среди гиперстеников частота людей с повышенным неустойчивым содержанием сахара выше, чем у нормо- и астеников. Последующие изменения скорости метаболизма и колебания гормонального статуса у людей с разным типом конституции в зрелом и пожилом возрасте могут быть сопряжены с выраженными колебаниями массы тела, увеличением нагрузки на диафрагму, изменение объема и протяженности кровеносного русла и других изменений. В результате происходят изменения геометрии и ориентации сердца в грудной полости. В этой связи определенный научный интерес представляет изучение динамики ЭОС у здоровых людей в связи с изменением их соматометрического статуса на протяжении разных периодов жизни.

Целью исследования являлось определение возрастных особенностей в динамике ЭОС у людей с разным соматометрическим статусом в пяти возрастных группах: дети дошкольного возраста от 4 до 7 лет, дети школьного возраста от 7 до 17 лет, взрослые от 21 до 35 лет (І период зрелого возраста), от 36 до 60 лет (II период зрелого возраста) и взрослые старше 60 лет.

\section{Материалы и методы.}

Для определения возрастной динамики ЭОС и оценки зависимости этого показателя от типа телосложения использовались результаты обследования лиц разного пола и возраста, проводимые за период 2015-2018 гг. на базе ГУЗ «Октябрьская больница» и лаборатории кафедры зоологии, физиологии и генетики УО «ГГУ имени Ф. Скорины». В обследовании приняли участие 375 человек, из них данные 264 человека использовались для проведения классификации и разработки кластерной модели соматотипов. Из общей выборки отбирались люди с выраженными особенностями телосложения, и значением индекса крепости телосложения, позволяющим определить принадлежности к тому или иному соматотипу (не менее 3-х человек в каждой возрастной группе). Измерение показателей ЭКГ проводились на оборудовании типа Интекард-3 и Альтоник-6 в соответствие с МУ; Рег. удостоверение № ИМ-7.6566/1604 ТУ ВY100050381.001-2005. При проведении обследования проводилась запись протокола, куда вносили данные о возрасте, результатах измерения длины, массы тела, окружности грудной клетки на вдохе и результатах ЭКГ анализа. Определение типа соматической конституции проводилось по методу Черноруцкого (Черноруцкий М.В., 1949 Чтецов В.П., 1972), который может быть использован в качестве количественной характеристики соматотипа для разных возрастных групп $[7,8]$. В основе метода лежит визуальная оценка и расчет индекса Пинье (показателя крепости телосложения). Согласно значениям индекса участники были распределены в три группы: астеники (больше 30), нормостеники (10 - 30) и гиперстеники (меньше 10). 
Массив данных разделен на группы в соответствие с выбранной нами градацией: дети дошкольного возраста от 4 до 7 лет, дети школьного возраста от 7 до 17 лет, взрослые от 21 до 35 лет (I период зрелого возраста), от 36 до 60 лет (II период зрелого возраста) и взрослые старше 60 лет. Выбранные периоды жизни захватывают время, в течение которого происходят наиболее выраженные изменения компонентов тела. $\mathrm{y}$ детей относительное развитие костного и мышечного компонента уступает жировому компоненту. У европеоидных мальчиков минимальные значения жироотложения наблюдаются в возрасте 8 лет, и достигает максимума к 12-13 годам (около 9 кг), после чего происходит падение жироотложения. У девочек наименьшие показатели роста жироотложения приходятся на 7 лет, но сам процесс продолжаются вплоть до 17 лет. К 21-ти годам устанавливается определенный ростовой баланс, где мышечная масса достигает наибольшего развития. В последующий период ближе к 30-ти годам происходит относительное уменьшение мышечного компонента и увеличение жирового. Особенно яркие изменения проявляются после 60 лет, когда начинает снижаться и жировой компонент тела (Морфология человека, 1990) [8].

Достоверности различий оценивалась исходя из нормального распределения значений соматометрических показателей и данных ЭКГ анализа на основе t-критерия Стьюдента. Для проведения анализа использовались методы регрессионного, кластерного и дискриминатного анализа. Кластерная модель разработана по аналогии модели [9]. Статистическая обработка результатов исследования выполнена с помощью прикладных программ MS Office Excel 2007 и Statistica for Windows 6.0 [10].

\section{Результаты исследования и обсуждение.}

В результате обследования получены данные позволившие рассчитатьсредние значения основных показателей соматометрии (длина, масса тела и окружность грудной клетки) у лиц мужского и женского пола в разные возрастные периоды. В таблице 1 приведены усредненные данные показателей соматометрии для лиц мужского пола.

Таблица 1

Возрастные показатели соматометрии лиц мужского пола

\begin{tabular}{|c|c|c|c|}
\hline $\begin{array}{c}\text { Возрастная } \\
\text { группа }\end{array}$ & $\begin{array}{c}\text { Длина } \\
\text { тела, см }\end{array}$ & Масса тела, кг & ОГК, см \\
\hline от 4 до 7 лет & $119,5 \pm 4,4$ & $25,2 \pm 2,4$ & $58,8 \pm 2,2$ \\
\hline от 7 до 17 лет & $155,9 \pm 1,9$ & $48,1 \pm 1,5$ & $74,7 \pm 0,9$ \\
\hline от 21 до 35 лет & $175,8 \pm 1,3$ & $72,4 \pm 2,1$ & $95,1 \pm 0,8$ \\
\hline от 36 до 60 лет & $176,8 \pm 0,9$ & $83,3 \pm 2,4$ & $92,5 \pm 1,4$ \\
\hline старше 60 лет & $170,6 \pm 0,7$ & $75,9 \pm 4,9$ & $88,9 \pm 9,2$ \\
\hline
\end{tabular}

Из таблицы 1 видно, что наибольшая вариация длинны тела, наблюдается у детей и подростков в возрасте 7 - 17 лет $12 \%$, массы тела - $27 \%$, у взрослых мужчин внутригрупповые вариации этих показателей не превышает $10 \%$.Минимальные вариация длинны тела наблюдается у мужчина старше 21 года и снижается к пожилому возрасту. Наибольшие значения вариации окружности грудной клетки наблюдаются в возрасте 7 - 17 лет и старше 60 лет - более $12 \%$, минимальная вариация этого показателя наблюдается в первого периода зрелости - $4 \%$. Сравнительный анализ показателей соматометрии показал достоверные различия между тремя возрастными группами -4 - 7 лет, 7 - 17 лет и группой старше 21 ( $<<0,05)$. Группы первого, второго периода зрелости и пожилого возраста не показали достоверного различия по данным показателям $(\mathrm{p}>0,05) . Н \mathrm{a}$ основании данныхсоматометрии для каждой возрастной группы были рассчитаны значения индекса крепости телосложения (индекс Пинье) и индекс массы тела (индекс Кетле). На рисунке 1 представлена возрастная динамика средних значений индекса крепости телосложения и массы тела.

Из рисунка 1 видно, что прослеживается четкая тенденция снижения с возрастом индекса Пинье и увеличение индекса Кетле. Полученная закономерность указывает на перераспределение количества мужчин из общей выборке в направление от большего количества с астеническим типом телосложения в период детства, в сторону нормостении (в зрелом возрасте), а затем гиперстении во ІІпериоде зрелого и пожилом возрасте. Коэффициент наклона линейной регрессия для индекса крепости телосложения составил - 7,2, коэффициент детерминации $0,82 \quad(\mathrm{p}<0,05)$. Коэффициент наклона линейной регрессия для индекса крепости 


\begin{tabular}{|c|c|c|c|c|c|c|}
\hline Impact Factor: & $\begin{array}{l}\text { ISRA (India) } \\
\text { ISI (Dubai, UAE } \\
\text { GIF (Australia) } \\
\text { JIF }\end{array}$ & $\begin{array}{l}=1.344 \\
=0.829 \\
=0.564 \\
=1.500\end{array}$ & $\begin{array}{l}\text { SIS (USA) } \\
\text { PИНЦ (Russia } \\
\text { ESJI (KZ) } \\
\text { SJIF (Moroccc }\end{array}$ & $\begin{array}{l}=0.912 \\
=0.156 \\
=4.102 \\
=\mathbf{5 . 6 6 7}\end{array}$ & $\begin{array}{l}\text { ICV (Poland) } \\
\text { PIF (India) } \\
\text { IBI (India) }\end{array}$ & $\begin{array}{l}=6.630 \\
=1.940 \\
=4.260\end{array}$ \\
\hline
\end{tabular}

телосложения составил $+2,1$, коэффициент детерминации $0,92(\mathrm{p}<0,05)$.

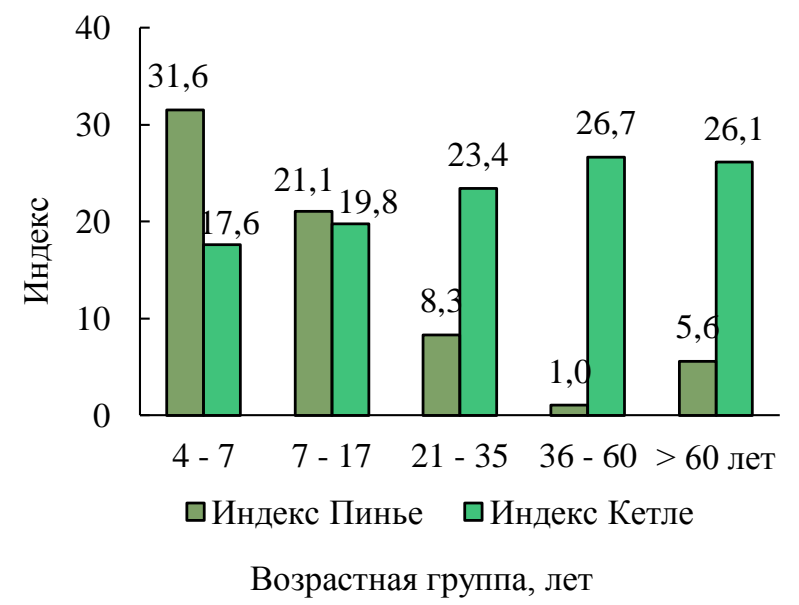

Рисунок 1 - Динамика индексов крепости телосложения и массы тела мужчин

В каждой возрастной группе были получены распределение индекса крепости телосложения, и в соответствие с методом Черноруцкого определены и отобраны по три человека с характерными признаками каждого соматотипа. В таблице 2 приведены средние значения показателей соматометрии астеников, нормо- и гиперстеников. В результате анализа данных в возрастной группе 36 - 60 и старше 60 лет мужчин астенического типа телосложения не оказалось.

\section{Соматометрия мужчин с разным типом телосложения}

Таблица 2

\begin{tabular}{|c|c|c|c|}
\hline $\begin{array}{c}\text { Возрастная } \\
\text { группа }\end{array}$ & $\begin{array}{c}\text { Длина } \\
\text { тела, см }\end{array}$ & $\begin{array}{c}\text { Масса } \\
\text { тела, кг }\end{array}$ & ОГК, см \\
\hline \multicolumn{4}{|c|}{ Астенический тип } \\
\hline 4 - 7 лет & $116,2 \pm 5,2$ & $18,5 \pm 2,4$ & $55,4 \pm 1,7$ \\
\hline 7 - 17 лет & $149,8 \pm 5,2$ & $38,4 \pm 3,7$ & $67,4 \pm 2,5$ \\
\hline 21 - 35 лет & $173,2 \pm 0,9$ & $62,6 \pm 2,6$ & $80,0 \pm 2,8$ \\
\hline 36 - 60 лет & - & - & - \\
\hline$>60$ лет & - & - & - \\
\hline \multicolumn{4}{|c|}{ Нормостенический тип } \\
\hline 4 - 7 лет & $100,5 \pm 6,6$ & $18,8 \pm 1,7$ & $54,5 \pm 3,8$ \\
\hline 7 - 17 лет & $155,6 \pm 6,8$ & $55,5 \pm 3,8$ & $79,5 \pm 2,6$ \\
\hline 21 - 35 лет & $177,9 \pm 1,3$ & $69,4 \pm 0,6$ & $91,9 \pm 2,8$ \\
\hline 36 - 60 лет & $176,9 \pm 2,4$ & $71,3 \pm 3,5$ & $92,0 \pm 2,5$ \\
\hline$>60$ лет & $172,0 \pm 0,8$ & $68,0 \pm 6,4$ & $82,0 \pm 4,6$ \\
\hline \multicolumn{4}{|c|}{ Гиперстенический тип } \\
\hline 4 - 7 лет & - & - & - \\
\hline 7 - 17 лет & $148,9 \pm 13,9$ & $58,6 \pm 5,8$ & $85,2 \pm 7,8$ \\
\hline 21 - 35 лет & $174,5 \pm 3,9$ & $77,6 \pm 5,9$ & $96,3 \pm 3,5$ \\
\hline 36 - 60 лет & $176,8 \pm 1,9$ & $85,3 \pm 3,9$ & $100,5 \pm 4,1$ \\
\hline$>60$ лет & $170,0 \pm 1,5$ & $82,2 \pm 4,4$ & $102,2 \pm 0,5$ \\
\hline
\end{tabular}

В таблице 4 приведены усредненные данные показателей соматометрии лиц женского пола. Сравнение коэффициентов вариации между возрастными группами показало, что, как и в случает мужчин, наибольшая вариации показателей соматометрии наблюдается в возрасте 


\begin{tabular}{|c|c|c|c|c|c|c|}
\hline Impact Factor: & $\begin{array}{l}\text { ISRA (India) } \\
\text { ISI (Dubai, UAE } \\
\text { GIF (Australia) } \\
\text { JIF }\end{array}$ & $\begin{array}{r}=1.344 \\
=0.829 \\
=0.564 \\
=1.500\end{array}$ & $\begin{array}{l}\text { SIS (USA) } \\
\text { PИHЦ (Russia) } \\
\text { ESJI (KZ) } \\
\text { SJIF (Morocco) }\end{array}$ & $\begin{array}{l}=0.912 \\
=0.156 \\
=4.102 \\
=\mathbf{5 . 6 6 7}\end{array}$ & $\begin{array}{l}\text { ICV (Poland) } \\
\text { PIF (India) } \\
\text { IBI (India) }\end{array}$ & $\begin{array}{l}=6.630 \\
=1.940 \\
=4.260\end{array}$ \\
\hline
\end{tabular}

7 - 17 лет, где значения этого показателя для длины тела составило - $11 \%$, для массы тела $26 \%$,для окружности грудной клетки $-13 \%$. Сравнительный анализ показателей соматометрии женщин показал достоверные различия длины тела между всеми возрастными группами $(\mathrm{p}<0,05)$, кроме группы $21-35$ и $36-60$ лет $(\mathrm{p}>0,05)$.

Возрастные показатели соматометрии лиц женского пола

Таблица 3

\begin{tabular}{|c|c|c|c|}
\hline $\begin{array}{c}\text { Возрастная } \\
\text { группа }\end{array}$ & $\begin{array}{c}\text { Длина } \\
\text { тела, см }\end{array}$ & $\begin{array}{c}\text { Масса } \\
\text { тела, кг }\end{array}$ & ОГК, см \\
\hline от 4 до 7 лет & $116,0 \pm 3,8$ & $25,9 \pm 3,2$ & $56,0 \pm 2,5$ \\
\hline от 7 до 17 лет & $148,5 \pm 3,2$ & $45,3 \pm 2,5$ & $72,2 \pm 1,9$ \\
\hline от 21 до 35 лет & $166,3 \pm 1,0$ & $57,9 \pm 2,8$ & $84,4 \pm 0,7$ \\
\hline от 36 до 60 лет & $164,0 \pm 1,5$ & $65,8 \pm 1,6$ & $91,3 \pm 1,8$ \\
\hline старше 60 лет & $157,6 \pm 0,2$ & $68,6 \pm 1,8$ & $102,4 \pm 1,2$ \\
\hline
\end{tabular}

На рисунке 2 представлена возрастная динамика средних значений индекса крепости телосложения и массы тела у женщин. Из рисунка видно, что в группе женщин тенденция снижения индекса крепости телосложения и увеличения массы тела не отличается от показанной выше динамики у мужчин. Вследствие чего можно сделать вывод об отсутствие каких-либо достоверных различий в возрастной динамике основных показателей соматометрии и наличие полового диморфизма.

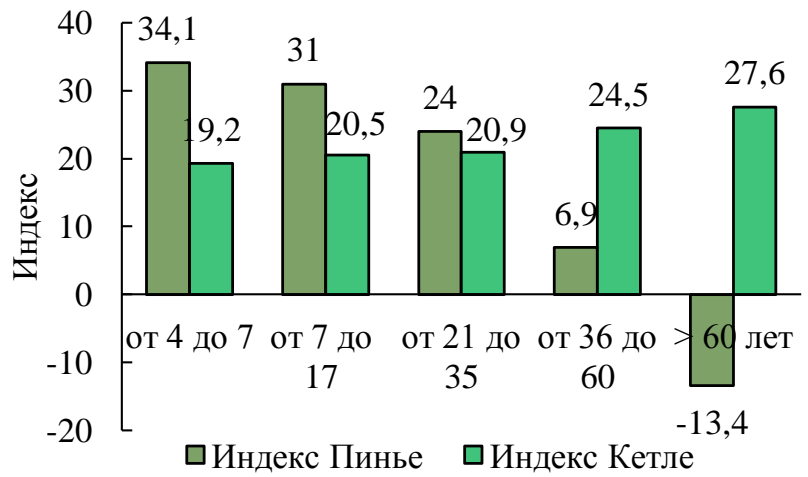

Возрастная группа, лет

\section{Рисунок 2 - Динамика индексов крепости телосложения и массы тела женщин}

Коэффициент наклона линейной регрессия для индекса крепости телосложения составил 12, коэффициент детерминации 0,91 ( $<<0,05)$. Коэффициент наклона линейной регрессия для индекса крепости телосложения составил +2 , коэффициент детерминации $0,91 \quad(\mathrm{p}<0,05)$. В данном случае обращает внимание больший угол наклона динамики индекса крепости телосложения у женщин в сторону гиперстенического типа. В таблице 5 приведены средние значения показателей соматометрии женщин астеников, нормо- и гиперстеников.

\section{Соматометрия женщин с разным типом телосложения}

Таблица 4

\begin{tabular}{|c|c|c|c|}
\hline $\begin{array}{c}\text { Возрастная } \\
\text { группа }\end{array}$ & $\begin{array}{c}\text { Длина } \\
\text { тела, см }\end{array}$ & $\begin{array}{c}\text { Масса } \\
\text { тела, кг }\end{array}$ & ОГК, см \\
\hline \multicolumn{4}{|c|}{ Астенический тип } \\
\hline $4-7$ лет & $121,7 \pm 5,3$ & $25,7 \pm 2,6$ & $57,6 \pm 2,2$ \\
\hline $7-17$ лет & $142,8 \pm 4,3$ & $36,8 \pm 2,7$ & $69,2 \pm 2,2$ \\
\hline $21-35$ лет & $169,9 \pm 2,8$ & $58,8 \pm 2,2$ & $82,6 \pm 0,5$ \\
\hline
\end{tabular}




\section{Impact Factor:}

\begin{tabular}{lr|lr} 
ISRA $($ India & $=\mathbf{1 . 3 4 4}$ & SIS $($ USA) & $=\mathbf{0 . 9 1 2}$ \\
ISI $($ Dubai, UAE) $=\mathbf{0 . 8 2 9}$ & PИНЦ (Russia) $=\mathbf{0 . 1 5 6}$ \\
GIF (Australia) $=\mathbf{0 . 5 6 4}$ & ESJI (KZ) & $=\mathbf{4 . 1 0 2}$ \\
JIF & $=\mathbf{1 . 5 0 0}$ & SJIF $($ Morocco $)=\mathbf{5 . 6 6 7}$
\end{tabular}

ICV (Poland)

$=6.630$

PIF (India)

$=1.940$ JIF

\begin{tabular}{|c|c|c|c|}
\hline $36-60$ лет & - & - & - \\
\hline$>60$ лет & - & - & - \\
\hline \multicolumn{5}{|c|}{ Нормостенический тип } \\
\hline $4-7$ лет & $112,4 \pm 6,1$ & $28,8 \pm 3,4$ & $58,9 \pm 3,7$ \\
\hline $7-17$ лет & $156,8 \pm 3,6$ & $51,6 \pm 3,1$ & $81,7 \pm 1,6$ \\
\hline $21-35$ лет & $165,6 \pm 1,6$ & $60,2 \pm 0,6$ & $84,7 \pm 0,9$ \\
\hline 36 - 60 лет & $165,6 \pm 1,7$ & $63,1 \pm 2,2$ & - \\
\hline$>60$ лет & - & - & $68,8 \pm 1,9$ \\
\hline \multicolumn{4}{|l|}{ Гиперстенический тип } \\
\hline 7 - 7 лет & $114,7 \pm 10,9$ & $36,2 \pm 3,5$ & $73,8 \pm 2,8$ \\
\hline $21-35$ лет & $129,8 \pm 6,9$ & $45,2 \pm 5,7$ & $94,7 \pm 1,3$ \\
\hline $36-60$ лет & $166,4 \pm 1,7$ & $65,3 \pm 1,8$ & $93,2 \pm 2,2$ \\
\hline$>60$ лет & $162,2 \pm 1,2$ & $68,2 \pm 3,6$ & $102,0 \pm 4,2$ \\
\hline
\end{tabular}

Статистический анализа данных, представленных в таблицах 2 и 4, а также расчетные значения показателей крепости телосложения и индекса массы тела в каждой группе подтвердил общую тенденцию, полученную на общей выборке - с возрастом в популяции мужчин и женщин происходит снижение индекса Пинье и увеличение индекса Кетле. Динамика этих процессов хорошо описывается уравнением линейной регрессией, где коэффициент смешанной корреляции превышает 0,9.

Используя методику кластерного анализа, установлено, что в общей выборке выделяются группы людей (3 кластера), значения показателей соматометрии которых имеют достоверное отличие $(\mathrm{p}<0,05)$. В первый кластер вошли типичные астеники, во второй - нормостеники, в третий - гиперстеники. На основании статистических характеристик каждого кластера методом дискриминантного анализа разработано правило классификации, позволяющее по данные обследования и учетом возраста определить принадлежность человека к одному из кластеров. Значения коэффициента Wilks' Lambda составило $0,09163$ approx. $F(8,516)=148,58 \mathrm{p}<0,001)$, что указывает на высокую точности дискриминации. Правило классификации принимает следующий вид (1-3):
$\mathrm{Y} 1=-0,85 \mathrm{~A}+0,95 \mathrm{H}-1,97 \mathrm{~W}+1,81 \mathrm{P}-88,9$

$\mathrm{Y} 2=-0,96 \mathrm{~A}+0,56 \mathrm{H}-1,30 \mathrm{~W}+2,28 \mathrm{P}-97,1$

$\mathrm{Y} 3=-0,85 \mathrm{~A}+0,28 \mathrm{H}-0,79 \mathrm{~W}+2,41 \mathrm{P}-92,9$

где A - возраст, полных лет; Н - длина тела, см; W - масса тела, кг; Р - окружность грудной клетки, cM.

Из кластерных ядер в возрастных группах были определены границы варьирования угла альфа электрической оси сердца, которые представлены в таблице 6 . К сожалению, таблица не заполнена полностью, поскольку исследование ограничено выборочными данными. Тем не менее, из данных представленных в таблице 6 видно, что значения угла электрической оси с возрастом смещается от первого кластера к третьему, как у мужчин, так и у женщин, т.е. от положения соответствующего правограмме к положению левограммы.

Из таблицы 6 видно, что положение угла наклона электрической оси сердца является характерной отличительной особенностью людей разного возраста и типа телосложения. Наиболее выражены эти отличия в детском и пожилом возрасте, где достоверная вероятность различия менее 0,05 .

Возрастные значения угла альфа ЭОС

Таблица 5

\begin{tabular}{|c|c|c|c|c|c|c|}
\hline \multirow[b]{3}{*}{ Группа } & \multicolumn{6}{|c|}{ ЭОС, градус } \\
\hline & \multicolumn{3}{|c|}{ Мужчины } & \multicolumn{3}{|c|}{ Женщины } \\
\hline & 1 & 2 & 3 & 1 & 2 & 3 \\
\hline $4-7$ & $83,1 \pm 3,5$ & - & - & $76,1 \pm 2,2$ & - & - \\
\hline $7-17$ & $57,5 \pm 4,3$ & $49,4 \pm 3,0$ & $37,1 \pm 2,1$ & $52,9 \pm 3,6$ & $49,8 \pm 1,0$ & $38,1 \pm 2,1$ \\
\hline $21-35$ & - & $45,3 \pm 1,4$ & $29,5 \pm 1,3$ & - & $42,2 \pm 3,0$ & $29,5 \pm 2,3$ \\
\hline
\end{tabular}




\begin{tabular}{|c|c|c|c|c|c|c|}
\hline $36-60$ & - & $46,3 \pm 2,4$ & $27,8 \pm 0,8$ & - & - & $28,0 \pm 1,9$ \\
\hline$>60$ & - & - & $25,5 \pm 2,3$ & - & - & $21,5 \pm 0,6$ \\
\hline
\end{tabular}

Достоверные различия между мужчинами и женщинами показаны в группе детей $4-7$ лет и людей старше 60 лет $(\mathrm{p}<0,05)$. Между мужчинами и женщинами нормостенического типа достоверные различия средних значений угла ЭОС не установлены $(\mathrm{p}>0,05)$. Полученные результаты полностью согласуется с литературными данными и позволяет сделать вывод о стойкой тенденции, которую обуславливают различия возрастных изменений у людей с разным соматотипом.

\section{Заключение.}

Полученные результаты свидетельствуют о наличие зависимости возрастного соматометрического статуса (т.е. возрастных особенностей соматотипа) и положения электрической оси сердца у людей разного пола и возраста. В результате исследования для практических целей разработана кластерная модель, которая позволяет без предварительного определения каких либо соматометрических индексов определить тип телосложения человека. Модель учитывает возрастные границы нормы не только показателей соматометрии того или иного типа, но и возрастную динамику угла наклона электрической оси сердца. Полученные с помощью кластерной модели соматотипов возрастные границы нормы значений угла наклона электрической оси сердца, могут быть использованы для сопоставления в качестве должных величин с данными ЭКГ-анализа. Однако следует отметить необходимость увеличения объема экспериментального материала, для уточнения полученных границ варьирования угла альфа и определения таковых в тех группа, где они не были определены.

\section{References:}

1. Zemcovskij, EH. V. (1995) Sportivnaya kardiologiya. - M.: Izd. Gippokrat. - 1995. $446 \mathrm{p}$.

2. Agadzhanyan M.G. (2005) EHlektrokardiograficheskie proyavleniya hronicheskogo fizicheskogo perenapryazheniya u sportsmenov / M. G. Agadzhanyan // Fiziologiya cheloveka. - 2005. - T. 31. - p. 6064.

3. Gavrilova E. A. (2010) Vnezapnaya serdechnaya smert' i gipertrofiya miokarda u sportsmenov / E. A. Gavrilova, EH. V. Zemcovskij // Vestn. aritmologii. - 2010. - T. 62, No 62. - p. $59-62$.

4. Garbusenko S.A. (2000) Geneticheskie aspekty gipertroficheskoj kardiomiopatii // Praktikuyushchij vrach. - 2000. - № 18. - p. 25.

5. Murashko, V.V., Strutynskij A.V. (1998) EHlektrokardiografiya: Ucheb. posobie. - M. :
OOO «MEDpress»; - EHlista. : APP «Dzhangar», 1998. - 313 p.

6. Zudnov, YU.I. (2003) Azbuka EHKG. Rostov-na-Donu : izd. Feniks, 2003. - p. 40-50.

7. Tegako, L. (2008) Antropologiya: uchebnoe posobie / L. Tegako, E. Kmetinskij. - M.: Novoe znanie, 2008. - p. 137

8. Nikityuk B.A. (1990) Morfologiya cheloveka / Pod red. B.A. Nikityuka, V.P. CHtecova. - M.: Izd - vo MGU, 1990. - 344 p.

9. Drozdov, D. N. (2008) Klasternaya model' optimizacii monitoringa DVO naseleniya, prozhivayushchego na territorii radioaktivnogo zagryazneniya / D. N. Drozdov // Izvestiya Gomel. gos. un-ta im. F. Skoriny. - 2008, № 5 (50) - p. 114-118.

10. Borovikov, V.P. (1998) Populyarnoe vvedenie v programmu STATISTICA: ucheb. posobie dlya stud. vyssh. ucheb. zavedenij / V.P. Borovikov. - M.: Komp'yuterPress, 1998. p.193. 